\title{
PRINCIPAL QUADRATIC FUNCTIONALS
}

\author{
BY \\ WALTER LEIGHTON(1)
}

This paper is a continuation of a study undertaken earlier by Marston Morse and the writer $[\mathrm{ML}, 1]\left({ }^{2}\right)$. While the present paper is essentially selfcontained, some familiarity on the part of the reader with the earlier paper, particularly with the importance of the singularity function in studying the minimizing of singular quadratic functionals when evaluated along $A$-admissible curves, is highly desirable. Classical Sturm separation and comparison theorems are used freely and usually without reference throughout the paper. The integrals employed in the analysis are Lebesgue integrals and their extensions.

The functional $J$ with which this paper has to do is termed a principal quadratic functional $(\$ 2)$. In general it is singular at $x=0$. It should be understood, however, that it is so defined as to include many nonsingular functionals as well. The function $p(x)$ which appears in $J$ is assumed to be of one sign near $x=0$. It will be seen that the Euler equation of $J$ thus includes, for example, all the classical singular second-order linear ordinary differential equations.

The variation of $J$ is studied both under fixed end conditions and when the $y$-axis is regarded as a kind of singular end curve with the second end point fixed. The concept of the focal point of the $y$-axis is introduced. Its theory characterizes precisely the variation of $J$ when $F$-admissible curves are the comparison curves. It is also found that the focal point of the $y$-axis rather unexpectedly plays a role in the study of the fixed end point problem (Theorem 4.1).

1. Introduction. Quadratic functionals of the type

$$
J_{1}=\int_{0}^{d} f\left(x, y, y^{\prime}\right) d x=\int_{0}^{d}\left[a(x) y^{\prime 2}+2 b(x) y y^{\prime}+c(x) y^{2}\right] d x
$$

where $a(x), b(x)$, and $c(x)$ are continuous functions of the real variable $x$ and $a(x)$ is positive on the interval $\left({ }^{3}\right)(0, h)(0<d<h)$ were studied systematically by Morse and Leighton $\left.{ }^{4}\right)[1]$. In that paper, as in this, a function $y(x)$ and

Presented to the Society, April 30, 1949; received by the editors August 20, 1948.

(1) The investigations of this paper were made in part while the author was under contract to the Office of Naval Research.

(2) Numbers in brackets refer to the bibliography at the end of the paper.

( $\left.{ }^{8}\right)$ In designating intervals it will be convenient to use the conventions: $[a, b]$ means the interval $a \leqq x \leqq b,(a, b]$ means the interval $a<x \leqq b,[a, b)$ means the interval $a \leqq x<b,(a, b)$ means the interval $a<x<b$.

(4) This paper will be referred to as ML. 
the curve $y=y(x)$ are said to be $A$-admissible on $[0, d]$ if

1. $y(x)$ is continuous on $[0, d]$ and $y(0)=y(d)=0$;

2. $y(x)$ is absolutely continuous and $y^{\prime 2}(x)$ is integrable Lebesgue on each closed subinterval of $(0, b]$.

The functional $J_{1}$ is said to possess a minimum limit on $[0, d]$ if for all $A$-admissible functions $y(x)$

$$
\lim \inf \int_{e}^{d} f\left(x, y, y^{\prime}\right) d x \geqq 0(5) .
$$

The Euler equation (written E.E.) associated with the functional $J_{1}$ is the differential equation

$$
\frac{d}{d x}\left(a y^{\prime}+b y\right)-\left(b y^{\prime}+c y\right)=0 .
$$

Clearly this equation may also be regarded as the Jacobi equation associated with $J_{1}$. A function $y(x)$ of class $C^{1}$ on the interval $(0, h)$ which satisfies (1.3) will be termed a solution. It will occasionally be convenient to refer to the corresponding curve $y=y(x)$ as a solution also.

The segment $[0, d]$ of the $x$-axis is $A$-admissible, and along this curve $J_{1}=0$. In establishing conditions under which (1.2) is valid Morse and Leighton were led to extend the concept of the first conjugate point on $[0, h)$ of the point $\left(^{6}\right) x=0$ to include the functional (1.1), and to introduce a new necessary condition (the singularity condition). This condition which is independent of the analogues of the classical Jacobi and Weierstrass conditions may be stated as follows. Let $u(x)$ be the solution of (1.3) such that $u(d)=0$, $u^{\prime}(d)=-1$. The singularity function $s[y, d]$ associated with the point $x=d$ and the $A$-admissible function $y(x)$ is defined by the relation

$$
s[y, d]=-y^{2}\left[a(x) \frac{u^{\prime}(x)}{u(x)}+b(x)\right] .
$$

Then, in order that (1.2) hold for all $A$-admissible functions $y(x)$ it is necessary that

$$
\liminf _{x \rightarrow 0+} s[y(x), d] \geqq 0
$$

for each $A$-admissible function $y(x)$ for which

$$
\liminf _{x \rightarrow 0+} \int_{x}^{d} f\left(x, y, y^{\prime}\right) d x
$$

(5) Unless specifically indicated otherwise, all limits taken in this paper will be limits as either $e$ or $x \rightarrow 0+$. It will be clear from the context which is intended.

(6) If will be convenient at times to refer to the point on the $x$-axis whose abscissa is $a$ as the point $x=a$. 
is finite. This condition is automatically satisfied by nonsingular quadratic functionals.

It is the expected phenomenon in the calculus of variations to find that the existence of a minimum depends on the class of functions admitted for comparison. Once the comparison curves have been selected it is, of course, desirable, when possible, to be able to state sufficient conditions for the existence of a minimum in terms of the "geometry" of the functional (for example, in terms of the behavior of the extremals, and so on). It will be seen that the singularity condition is not of this type requiring, as it does, that one verify that (1.4) hold for all $A$-admissible curves along which $\lim \inf J_{1}$ is finite. The conditions which are obtained in the present paper do not depend formally (that is, as stated) on the class of curves admitted. This is possible because the functional $J_{1}$ has been specialized moderately. A number of the results which have been obtained, as will be seen, depend fundamentally on the singularity condition. It is important that it be understood that when curves which are $A$-admissible are admitted for comparison in $J_{1}$, this type of condition must not be unexpected, partly because of the generality permitted the function $b(x)$. The following analysis will make this point clear.

Suppose for simplicity that $b(x)$ is of class $C^{1}$ on $(0, h)$. It is then easy to verify that

$$
\int_{e}^{d}\left[a y^{\prime 2}+2 b y y^{\prime}+c y^{2}\right] d x=\left.b y^{2}\right|_{e} ^{d}+\int_{e}^{d}\left[a y^{\prime 2}+\left(c-b^{\prime}\right) y^{2}\right] d x(0<e<d) .
$$

For brevity we write this relation as

$$
\left.J_{1}(y)\right|_{e} ^{d}=\left.b y^{2}\right|_{e} ^{d}+\left.J(y)\right|_{e} ^{d}
$$

The E. E. of $J_{1}$ may now be written in the form

$$
\frac{d}{d x}\left(a y^{\prime}\right)+\left(b^{\prime}-c\right) y=0
$$

which is also the E. E. of $J$. It is clear that if we regard the two functions $a(x)$ and $b^{\prime}(x)-c(x)$ as fixed, $J_{1}$ and $J$ will have the same E. E., the same Jacobi equations, and the same Weierstrass $E$-function $E(x, y, \lambda, \mu)=a(\lambda-\mu)^{2}$ independent of the definition of $b(x)$. Suppose then that conditions depending, say, only on the behavior of the extremals of $J$ (and hence of $J_{1}$ ) have been determined which will insure that $\left.\lim \inf J\right|_{e} ^{d} \geqq 0$ for all $A$-admissible curves. These conditions can never be sufficient to show that $\left.\lim \inf J_{1}\right|_{e} ^{d} \geqq 0$ for all $A$-admissible curves. For suppose $\lim J[y(x)]_{e}^{d}=k>0$ for some $A$-admissible curve $y=y(x)$ of class $C^{1}$ on $(0, h)$ which passes through the point $(d, 0)$ and which has no point in common with the $x$-axis on the interval $(0, d)$. Choose $b(x)=(k+1) / y^{2}(x)$ in the neighborhood of $x=0$ and define it arbitrarily else- 
where subject to the condition that $b(x)$ is class $C^{1}$ on $(0, h)$. Then by equation (1.5)

$$
\lim J_{1}[y(x)]_{0}^{d}=\lim \left[-b(e) y^{2}(e)+k\right]=-1 .
$$

It should be noted that if $b$ and $b^{\prime}$ are continuous on $[0, h)$, then $\left.\lim b y^{2}\right|_{e} ^{d}=0$ and $J=J_{1}$ for all curves for which either exists.

These observations indicate why one cannot hope to establish sufficient conditions that $\left.\lim \inf J_{1}\right|_{e} ^{d} \geqq 0$ for all $A$-admissible curves not only not in terms of the analogue of the classical conjugate point theory, but not even in terms of more general properties of the solutions of the Euler equation.

EXAMPLE 1.1. Let $a(x)=1, c(x)-b^{\prime}(x)=-1, d=1$. If $y=y(x)$ is any $A$-admissible curve, equation (1.5) becomes

$$
(1.5)^{\prime} \quad \int_{e}^{1}\left[y^{\prime 2}+2 b y y^{\prime}+\left(b^{\prime}-1\right) y^{2}\right] d x=-b(e) y^{2}(e)+\int_{e}^{1}\left(y^{\prime 2}-y^{2}\right) d x .
$$

The extremals of the first integral, like those of the last, are the functions $c_{1} \sin x+c_{2} \cos x$, where $c_{1}$ and $c_{2}$ are constants. It is known [ML, p. 267]

$$
\lim \inf \int_{e}^{1}\left(y^{\prime 2}-y^{2}\right) d x \geqq 0
$$

for all $A$-admissible curves, and this limit is zero only when $y=0$. If $y(x)$ $=x-x^{2}, y(x)$ is $A$-admissible, and

$$
\begin{aligned}
\lim J[y(x)]_{e}^{1} & =3 / 10, \\
\lim \inf J_{1}[y(x)]_{e}^{1} & =3 / 10-\lim \inf b(e)\left[e-e^{2}\right]^{2} .
\end{aligned}
$$

If in the neighborhood of $x=0, b(x)=13 / 10\left(x-x^{2}\right)^{2}$, this last limit has the value -1 .

The foregoing considerations lead us to study principal quadratic functionals which are defined below.

2. The principal quadratic functional. In what follows we specialize (1.1) and change the notation slightly as follows. We set $b(x)=0$ and write

$$
a(x)=r(x), \quad c(x)=-p(x), \quad d=b .
$$

Our functional then becomes

$$
J=\int_{0}^{b}\left[r(x) y^{\prime 2}-p(x) y^{2}\right] d x .
$$

We shall use the symbol $\left.J(y)\right|_{a} ^{c}$ (and obvious variants of it) to mean 


$$
\left.J(y)\right|_{a} ^{c}=\int_{a}^{c}\left[r(x) y^{\prime 2}-p(x) y^{2}\right] d x,
$$

where it will be understood that $0<a<c \leqq b$. We term (2.2) a principal quadratic functional. Its $\mathrm{E}$. $\mathrm{E}$. is

$$
\frac{d}{d x}\left(r y^{\prime}\right)+p y=0 .
$$

In order to facilitate our later analysis it will be helpful to have proved in advance a number of properties of solutions of the $E$. E. These results are presented in the lemmas which follow.

LEMMA 2.1. If $x=0$ is not its own first conjugate point and if $p(x) \not \equiv 0$ does not change sign near $\left(^{7}\right) x=0$, the derivative of a nonnull solution of the $E$. $E$. is of one sign for $x$ near zero.

Since $x=0$ is not its own first conjugate point every nonnull solution $y(x)$ of the E.E. is of one sign near $x=0$. Suppose the conclusion of the lemma is false. On every interval $(0, e)(e>0) y^{\prime}$, and hence $r y^{\prime}$, must then vanish infinitely often. Between any pairs of zeros of $r y^{\prime}$ its derivative $\left(r y^{\prime}\right)^{\prime}=-p y$ must vanish at least once. Then $p$ must vanish infinitely often near $x=0$, contrary to hypothesis. From this contradiction we infer the truth of the lemma.

It is easy to verify that if $u(x) \not \equiv 0$ is a solution of the E. E. such that $u^{\prime}\left(x_{0}\right)=0\left(0<x_{0}<b\right)$ and $u(a)=0$, then every solution $y(x)$ which is orthogonal to the line $x=x_{0}$ takes the form $y\left(x_{0}\right) u(x)$, and $y(a)=0$. Further, if $u(x)$ vanishes for some value of $x$ following $x_{0}$, there is a first zero $a$ of $u(x)$ following $x=x_{0}$. When the number $a$ exists we call it the focal point $\left({ }^{8}\right)$ of the line $x=x_{0}$. If $u(x) \neq 0$ for $x>x_{0}$, we say that the line $x=x_{0}$ has no focal point. This terminology is justified by the fact that when the end curve is a vertical line, transversality is equivalent to orthogonality for the functional $J$, as one may readily prove.

LEMMA 2.2. If the focal point $a$ of the line $x=x_{0}>0$ exists, then

$$
\frac{d a}{d x_{0}}=p\left(x_{0}\right)\left[\frac{r(a) v^{2}(a)}{r^{2}\left(x_{0}\right) v^{\prime 2}\left(x_{0}\right)}\right],
$$

where $v(x)$ is an arbitrary nonnull solution of the $E$. $E$.

To prove the lemma let $u(x)$ and $w(x)$ be linearly independent solutions of the E. E. Then

(7) The phrases "near $x=0$ " and " $x$ near zero" will be understood to mean $x$ (lies) on an interval $(0, e)$ where $e>0$ is taken suitably small.

(8) Cf. $\$ 4$ of this paper. For the classical theory of the focal point the reader is referred to Morse [1, p. 51]. 


$$
r(x)\left[u(x) w^{\prime}(x)-u^{\prime}(x) w(x)\right] \equiv c
$$

It follows that

$$
\frac{w^{\prime}(x)}{u^{\prime}(x)}-\frac{w(x)}{u(x)}=\frac{c}{r(x) u(x) u^{\prime}(x)} .
$$

It should be observed that there is no assurance that $u^{\prime \prime}(x)$ and $w^{\prime \prime}(x)$ exist and that the form of the proof does not require the existence of these second derivatives. An integration by parts indicates that

$$
\int_{e}^{x}\left[r u^{\prime 2}-p u^{2}\right] d x=\left.r u u^{\prime}\right|_{e} ^{x} \text {. }
$$

Since the left-hand member of (2.8) possesses a derivative, the right-hand member does also and

$$
\frac{d}{d x}\left(r u u^{\prime}\right)=r u^{\prime 2}-p u^{2}
$$

It follows from $(2.7)$ that $d / d x\left(w^{\prime} / u^{\prime}\right)$ exists and

$$
\frac{d}{d x}\left(\frac{w^{\prime}}{u^{\prime}}\right)=c\left[\frac{1}{r u^{2}}-\frac{r u^{\prime 2}-p u^{2}}{\left(r u u^{\prime}\right)^{2}}\right]=\frac{c p}{\left(r u^{\prime}\right)^{2}} .
$$

It follows from (2.10) that $w^{\prime} / u^{\prime}$ is of class $C^{1}$ except in the zeros of $u^{\prime}(x)$. A nonnull solution of the E. E. orthogonal to the line $x=x_{0}$ is $u^{\prime}\left(x_{0}\right) w(x)$ $-w^{\prime}\left(x_{0}\right) u(x)$. By hypothesis, its first zero $a$ following $x=x_{0}$ exists. Thus, if the particular solution $u(x)$ is such that $u^{\prime}\left(x_{0}\right) \neq 0$, then $u(a) \neq 0$ and $a$ is a solution of the equation

$$
\frac{w(a)}{u(a)}=\frac{w^{\prime}\left(x_{0}\right)}{u^{\prime}\left(x_{0}\right)} .
$$

Since $d / d a(w(a) / u(a))=c / r(a) u^{2}(a)$, which is nonzero and finite, the implicit function theorem [cf. Bliss 1, p. 269] applies to (2.11) and affirms the existence of $d a / d x_{0}$; consequently,

$$
\frac{d}{d a}\left(\frac{w(a)}{u(a)}\right) \frac{d a}{d x_{0}}=\frac{d}{d x_{0}}\left(\frac{w^{\prime}\left(x_{0}\right)}{u^{\prime}\left(x_{0}\right)}\right)=\frac{c p\left(x_{0}\right)}{\left[r\left(x_{0}\right) u^{\prime}\left(x_{0}\right)\right]^{2}} .
$$

The last equality above follows from (2.10). Accordingly,

$$
\frac{d a}{d x_{0}}=p\left(x_{0}\right)\left[\frac{r(a) u^{2}(a)}{r^{2}\left(x_{0}\right) u^{\prime 2}\left(x_{0}\right)}\right]\left({ }^{9}\right) \text {. }
$$

(9) If $a$ were a conjugate point of $x_{0}$, the corresponding formula would then be $d a / d x_{0}=r(a)$ $\cdot u^{2}(a) / r\left(x_{0}\right) u^{2} W\left(x_{0}\right)$. 
The application of (2.11) to (2.12) shows that $u(a)$ and $u^{\prime}\left(x_{0}\right)$ may be replaced by $w(a)$ and $w^{\prime}\left(x_{0}\right)$, respectively. The "singularity" of $d a / d x_{0}$ induced by the possibility that $u^{\prime}\left(x_{0}\right)=0$ is thus seen to be "removable." In fact, the ratio

$$
\frac{u(a)}{u^{\prime}\left(x_{0}\right)} \equiv \frac{w(a)}{w^{\prime}\left(x_{0}\right)}
$$

is seen to be an invariant of $J$, when the fraction is assigned a suitable value at the "singular" value $x=x_{0}$ and $a$ is understood to be the focal point of the line $x=x_{0}$. This observation concludes the proof of the lemma.

One notes that the bracket in (2.5) is continuous in $x_{0}$ and is positive, when it is defined properly at points $a$ and $x_{0}$ at which, respectively, a particular solution $v(x)$ may have the properties $v(a)=0, v^{\prime}\left(x_{0}\right)=0$.

These remarks lead to the following result.

Lemma 2.3. If the focal point a of a line $x=x_{0}$ exists, a decreases steadily as $x_{0}$ decreases in domains in which $p(x)>0$ and increases steadily as $x_{0}$ decreases in domains where $p(x)<0$.

The proof is trivial.

LEMMA 2.4. If $p(x)>0$ for $x$ near zero, if $x=0$ is not its own first conjugate point, and if for a positive and sufficiently small the solution $u(x, a)$ of the $E$. E. determined by the conditions

$$
u(a, a)=0, \quad u_{x}(a, a)=-1
$$

has the property that $u_{x}\left(x_{0}, a\right)=0$ for some number $x_{0}$ on $(0, a)$, then if $a$ is taken small enough, $x_{0}$ is uniquely determined and $u_{x}(x, a)>0$ for $x$ on $\left(0, x_{0}\right)$.

First of all, if for every $a>0$ there were more than one number on $(0, a)$ at which $u_{x}(x, a)=0$, this would contradict Lemma 2.1 . Thus $x_{0}$ is uniquely determined for $a$ small and $a$ is the focal point of the line $x=x_{0}$. It follows that for $a$ small $u(x, a)>0$ on $(0, a)$. We suppose now that $a$ is also taken small enough that $p(x)>0$ on $(0, a)$. Then $u_{x}(x, a)<0$ on $\left(x_{0}, a\right)$ and is of fixed sign on $\left(0, x_{0}\right)$. Suppose $u_{x}<0$ on $\left(0, x_{0}\right)$. Through a point $x=c>0$ preceding but near $x=x_{0}$ on the curve $y=u(x, a)$ construct a solution $v(x)$ of the E. E. such that $v^{\prime}(c)=0$. By elementary Sturm theory the first zero of $v(x)$ must follow $x=a$. By Lemma 2.2 it must precede $x=a$. From this contradiction we infer that $u_{x}(x, a)>0$ on $\left(0, x_{0}\right)$. The proof of the lemma is complete.

We return to our study of the principal quadratic functional. It will be clear in the sequel that we shall be able to state sufficient conditions for the existence of a minimum limit for these functionals in terms of the geometry of $J$ (although not in terms of the extended conjugate point theory alone).

We introduce a principal theorem. 
TheOREM 2.1. If $p(x)>0$ when $x$ is near zero and if

$$
\lim \int_{x}^{b} p(x) d x=+\infty,
$$

the functional $J$ does not possess a minimum limit.

This theorem is a generalization of several results proved earlier [ML, pp. $272 \mathrm{ff}$.]. To prove the theorem we shall construct an $A$-admissible curve along which $J$ assumes a finite negative value. The construction is similar to one used by ML [p. 272]. It has, however, the advantage of producing an $A$-admissible curve along which $J$ is surely finite.

We observe first that since $p(x)>0$ for $x$ near zero, there exists a unique solution $z_{n}(x)\left(x \leqq x_{n}\right)$ of the differential system

$$
\begin{array}{rlr}
r z^{2}-p z^{2} & =0, \\
z\left(x_{n}\right) & =y_{n} & \\
z^{\prime}(x) & >0 & \left(x_{n}>0, y_{n}>0, x_{n} \text { sufficiently small }\right), \\
& & \left(0<x \leqq x_{n}\right) .
\end{array}
$$

This solution can be written in the form

$$
z_{n}(x)=y_{n} \exp \int_{x_{n}}^{x}(p / r)^{1 / 2} d x
$$

Clearly, $\left.J\left(z_{n}\right)\right|_{e} ^{x_{n}}=0\left(0<e<x_{n}\right)$. Next we note that along curves $y=$ constant $\neq 0$ the functional $\left.J(y)\right|_{e} ^{c}(0<e<c)$ decreases without limit as $e \rightarrow 0$.

The curve is now constructed as follows. Starting at the point $(b, 0)$ we follow the line segment terminating at this point which has slope -1 and length $b$. Commencing at the initial point of this segment the curve follows a straight line segment parallel to the $x$-axis and directed to the left until an initial point $\left(x_{0}, y_{0}\right)$ is attained such that $p(x)>0$ on $\left(0, x_{0}\right)$ and $\left.J\right|_{x_{0}} ^{b}$ assumes a value $k<0$ along this broken curve. At this point the construction follows one of two possible courses according as the curve $y=z_{0}(x)$ does or does not pass through the origin $\left({ }^{10}\right)$. In the former case we complete the construction by following the curve $y=z_{0}(x)$ to the origin.

If the curve $y=z_{0}(x)$ does not pass through the origin the constructed curve is completed by a succession of arcs

$$
g_{0}, g_{1}, g_{2}, \cdots
$$

defined as follows. The arc $g_{n}$ terminates at the point $\left(x_{n}, y_{n}\right)(n=0,1,2, \cdots)$. When $n$ is even, take $x_{n+1}=x_{n} / 2$ and define $g_{n}$ by the relation $y=z_{n}(x)$ $\left(x_{n+1}<x \leqq x_{n}\right)$. For $n$ odd $g_{n}$ is the curve $y=y_{n}\left(x_{n+1}<x \leqq x_{n}\right)$, where $x_{n+1}$ is chosen such that $J[y]_{x_{n+1}}^{x_{n}}=k / 2^{n}$. Writing the equation of this curve as $y=y(x)$

(10) Throughout this paper we shall say that a curve $y=y(x)$ passes through the origin if $\lim y(x)=0$. 
we complete the definition of the curve by defining $y(0)=0$.

In both cases the curve is $A$-admissible. Along the former $J$ assumes the value $k<0$. Along the latter $J=2 k<0$.

The proof of the theorem is complete.

We continue with the following statement.

TheOREM 2.2. If $p(x)>0$ for $x$ near $x=0$, if there is no point on $[0, b)$ conjugate to $x=0$, and if

$$
\lim \int_{x}^{b} p(x) d x
$$

exists and is finite, the functional $J$ possesses a minimum limit.

We first recall [ML, p. 256] that since $x=0$ is not its own first conjugate point there exists a solution $w(x)$ of the E. E. with the property that its positive zeros (if any) are the conjugate points of $x=0$ and such that

$$
\lim \frac{w(x)}{u(x)}=0
$$

where $u(x)$ is any extremal which is linearly independent of $w(x)$. Such a solution $w(x)$ will be called a principal solution of the E. E.

To prove Theorem 2.2 we shall employ two lemmas.

LEMMA 2.5. If $x=0$ is not its own first conjugate point, if $p(x)>0$ near $x=0$, and if all solutions of the E. E. pass through the origin, then

$$
\lim \frac{w^{\prime}(x)}{u^{\prime}(x)}=0,
$$

where $u(x)$ is any extremal linearly independent of a principal solution $w(x)$.

We note first that since $x=0$ is not its own first conjugate point we may assume without loss in generality that $w(x)$ and $u(x)$ are positive for $x$ near zero. The one-parameter family of solutions through the point $x=a(0<a<b)$ may be written in the form

$$
y=c[u(x) w(a)-w(x) u(a)] .
$$

By an application of Rolle's theorem

$$
u^{\prime}\left(x_{0}\right) w(a)-w\left(x_{0}\right) u(a)=0,
$$

where $x_{0}$ is some point on $(0, a)$. For $a$ near zero, $u(a) \neq 0$ and $u^{\prime}\left(x_{0}\right) \neq 0$, and thus

$$
\frac{w^{\prime}\left(x_{0}\right)}{u^{\prime}\left(x_{0}\right)}=\frac{w(a)}{u(a)} .
$$


For $a$ near zero, $x_{0}$ is uniquely defined by $a$ and decreases steadily as $a$ decreases [Lemmas 2.3 and 2.4]. Since $\lim _{a \rightarrow 0+} w(a) / u(a)=0,(2.16)$ follows as stated.

We remark that the hypothesis that all solutions of the E. E. pass through the origin may be replaced by the condition that all solutions of the E. E. which are positive near $x=0$ have positive slopes near $x=0$.

Lемма 2.6. If the hypotheses of the preceding lemma hold, then

$$
\lim \int_{x}^{b} p(x) d x=+\infty .
$$

To prove this lemma observe first that a principal extremal $w(x)$ and a linearly independent extremal $u(x)$ may be chosen so that both are positive for $x$ near zero and such that

$$
r\left(u w^{\prime}-u^{\prime} w\right) \equiv 1 .
$$

It follows that

$$
\frac{w^{\prime}}{u^{\prime}}-\frac{w}{u}=\frac{1}{r u u^{\prime}} .
$$

Since the left-hand member of (2.19) tends to zero as $x$ tends to zero so does the right-hand member. Since $p(x)>0$ for $x$ near zero, $u^{\prime}(x)$ is of fixed sign for $x$ near zero. Since $u(x)>0$ and tends to zero, $u^{\prime}(x)>0$ for $x$ near zero. Thus $r u u^{\prime} \rightarrow+\infty$. It follows that $r u^{\prime} \rightarrow+\infty$. But from the E. E. we have( $\left.{ }^{11}\right)$

$$
r(x) u^{\prime}(x)=r(b) u^{\prime}(b)+\int_{x}^{b} p(x) u(x) d x,
$$

and, consequently, the integral in (2.20) becomes positively infinite.

We remark that the conclusion of the lemma would remain valid if we weakened the hypotheses and required simply that all solutions of the E. E. which are positive near $x=0$ have positive derivatives near $x=0$.

We return to the proof of Theorem 2.2. By a result due to ML $[1$, p. 264] it will be sufficient to show that the singularity condition is satisfied for all $A$-admissible curves $y=y(x)$. For $J$ the singularity condition is

$$
\lim \inf \left[-r(x) \frac{v^{\prime}(x)}{v(x)} y^{2}(x)\right] \geqq 0,
$$

where $v(x)$ may be taken as any solution $\not \equiv 0$ of the E. E. such that $v(b)=0$. We define $v(x)$ by the further condition that $v^{\prime}(b)=-1$.

We identify two cases according as $x=b$ is not or is the first conjugate point of $x=0$.

(11) It should be observed that equation (2.20) is valid for all solutions $u(x)$ of the E. E. 
Case I. First suppose $x=b$ is not conjugate to $x=0$. Then $v(x)$ is a nonprincipal solution of the E. E. which is positive on $(0, b)$. Since $p(x)>0$ near $x=0, v^{\prime}(x)$ is of fixed sign near $x=0$. Therefore, $\lim v(x)$ exists, finite or infinite, and is not negative. But $\lim v(x) \neq 0$; otherwise, $\lim w(x)=0$, also, and all solutions would go through the origin which would contradict Lemma 2.6. If $v^{\prime}(x)<0$ near $x=0,(2.21)$ clearly holds. Suppose then that $v^{\prime}(x)>0$. Then $\lim v(x)$ is finite and positive. By the hypotheses of our theorem and equation (2.20) $\lim r(x) v^{\prime}(x)$ exists and is finite. Accordingly,

$$
\lim \left[-r(x) \frac{v^{\prime}(x)}{v(x)} y^{2}(x)\right]=0 .
$$

Thus (2.21) is established if $x=b$ is not conjugate to $x=0$.

Case II. Finally, suppose $x=b$ is conjugate to $x=0$. In this case $v(x)$ is a principal solution. One observes that the foregoing analysis applies mutatis mutandis to this case except possibly when the following conditions hold (simultaneously):

$$
\begin{array}{rlrl}
v(x) & >0 & & (x \text { near zero }), \\
v^{\prime}(x) & >0 & & (x \text { near zero }), \\
\lim v(x) & =0 . &
\end{array}
$$

If $u(x)$ is a solution linearly independent of $v(x), \lim u(x) \neq 0$. Thus Theorem 5.3 of ML applies, since $J$ possesses a minimum limit on a shorter interval $[0, b-e]$ according to Case $\mathrm{I}$.

The proof of Theorem 2.2 is complete.

Corollary 2.1. If $p(x)>0$ near $x=0$, and if all solutions of the E. E. pass through the origin, the functional $J$ does not possess a minimum limit.

The corollary is seen to be an immediate consequence of the foregoing analysis.

Theorem 2.3. If $p(x)>0$ near $x=0$, if there is no point conjugate to $x=0$ on the interval $[0, b)$, and if there is a solution $v(x)$ of the $E$. E. such that $\lim u(x)$ $=\infty, J$ possesses a minimum limit.

Case I. Suppose $x=b$ is not conjugate to $x=0$. The solution $v(x)$ defined by the conditions $v(b)=0, v^{\prime}(b)=-1$ is a nonprincipal solution; consequently, $\lim v(x)=+\infty$. Since $v^{\prime}(x)$ cannot change sign near $x=0, v^{\prime}(x)<0$ near $x=0$, and the singularity condition is satisfied.

Case II. Suppose $x=b$ is conjugate to $x=0$. According to Case $I, J$ possesses a minimum limit over a somewhat shorter interval $[0, b-e]$. Theorem 5.3 of ML then affirms that $J$ possesses a minimum limit over $[0, b]$.

The following result is added for completeness.

THEOREM 2.4. If $p \leqq 0$ near $x=0$, and if there is no point on $[0, b)$ conjugate 
to $x=0, J$ possesses a minimum limit.

This is Theorem 5.4 of ML (with the notation slightly changed).

We introduce the following result which provides content to the first construction in the proof of Theorem 2.1.

THEOREM 2.5. If $p(x)>0$ for $x$ near zero, if $x=0$ is not its own first conjugate point, and if all solutions of the E. E. pass through the origin, the solution

$$
y(x)=y_{0} \exp \int_{x_{0}}^{x}(p / r)^{1 / 2} d x \quad\left(x_{0}>0, y_{0}>0\right)
$$

of the differential equation $r y^{\prime 2}-p y^{2}=0$ passes through the origin.

Let $u(x)$ be a solution of the E. E. which is linearly independent of a principal solution $w(x)$. As in the proof of Lemma 2.6 one can argue that $\lim r u u^{\prime}=+\infty$. Then $\left(r u u^{\prime}\right)^{\prime}=r u^{\prime 2}-p u^{2} \rightarrow-\infty$. It follows that

$$
\frac{p u^{2}-r u^{\prime 2}}{r u^{2}}>0
$$

for $x$ near zero. Thus for these values of $x$ it follows successively that if $x_{0}$ is a sufficiently small positive constant

$$
\begin{gathered}
(p / r)^{1 / 2}>u^{\prime} / u>0, \\
\int_{x}^{x_{0}}(p / r)^{1 / 2} d x>\int_{x}^{x_{0}} u^{\prime} / u d x>0, \\
y_{0} \exp \int_{x}^{x_{0}}(p / r)^{1 / 2} d x>y_{0} u\left(x_{0}\right) / u(x)>0,
\end{gathered}
$$

and hence

$$
0<y(x)<u(x) / y_{0} u\left(x_{0}\right) \quad\left(0<x<x_{0}\right) .
$$

The conclusion of the theorem is a consequence of (2.22).

The following example demonstrates that the conditions $p(x)>0$ for $x$ near zero, and $\lim \int_{x}^{b} p(x) d x=+\infty$ do not imply that all extremals of $J$ pass through the origin.

EXAMPLE 2.1. It is easy to verify that if

$$
r(x)=\frac{1}{2+x^{1 / 2}}, \quad p(x)=\frac{1}{2 x^{3 / 2}\left(2+x^{1 / 2}\right)^{2}}
$$

then linearly independent extremals are $w(x)=x$ and $u(x)=1+x^{1 / 2}$. It will be noted that in this case

$$
z(x)=c\left[x^{1 / 4}+\left(2+x^{1 / 2}\right)^{1 / 2}\right] 2^{3 / 2},
$$


where $c>0$ is constant.

We next exhibit two examples in which $p(x)$ is not of one sign near $x=0$. In both cases the minimum limits of $J$ exist. In the former $\lim \int_{x}^{b} p(x) d x$ exists, while in the latter this limit does not exist.

EXAMPLE 2.2. If

$$
r(x)=e^{-1 / 2 x^{2}}, \quad p(x)=\frac{\cos 1 / x-2 x}{x^{5}(2+\sin 1 / x)} e^{-1 / 2 x^{2}},
$$

the functional

$$
J=\int_{0}\left(r y^{\prime 2}-p y^{2}\right) d x
$$

has as a principal solution of its E. E.

$$
w(x)=x(2+\sin 1 / x) .
$$

Thus there is no conjugate point of $x=0$ on $[0,1]$. A linearly independent solution is

$$
u(x)=w(x) \int_{x}^{1} \frac{e^{1 / 2 x^{2}}}{x^{2}(2+\sin 1 / x)^{2}} d x=w(x) I(x) .
$$

One observes that $u(1)=0$. It is clear that $J$ exists along all $A$-admissible curves. The singularity function takes the form

$$
-r \frac{u^{\prime}}{u} y^{2}=\left[\frac{1}{x^{2}(2+\sin 1 / x)^{2} I(x)}-\frac{w^{\prime}}{w} e^{-1 / 2 x^{2}}\right] y^{2},
$$

which tends to zero as $x$ tends to zero. Thus $J$ possesses a minimum limit. In this example $\int_{0}^{1} p(x) d x$ exists.

EXAMPLE 2.3. If

$$
r(x)=x^{2}, \quad p(x)=\frac{\sin 1 / x}{x^{2}(2+\sin 1 / x)},
$$

linearly independent solutions of the E. E. associated with

$$
J=\int_{0}^{1}\left(r y^{\prime 2}-p y^{2}\right) d x
$$

are

$$
w(x)=2+\sin 1 / x, \quad u(x)=w(x) \int_{x}^{1} \frac{d x}{x^{2}(2+\sin 1 / x)^{2}}=w(x) I(x) .
$$

The singularity function for $J$ is 


$$
-r \frac{u^{\prime}}{u} y^{2}=\left[\frac{1}{(2+\sin 1 / x)^{2} I(x)}+\frac{\cos 1 / x}{2+\sin 1 / x}\right] y^{2},
$$

which tends to zero for every $A$-admissible function $y(x)$. Since $w(x)>0$ for all $x>0$, there are no points conjugate to $x=0$, and $J$ possesses a minimum limit. In this example $\int_{0}^{1} p(x) d x$ fails to exist.

We conclude with the remark that if $b(x)$ is of class $C^{1}$ on $[0, h)$, or if $b(x)$ is $C^{1}$ on $(0, h)$ and

$$
\lim b(x) y^{2}(x)=0
$$

for all $A$-admissible functions $y(x)$, the results of this section apply to the functional $J_{1}$ of (1.1). One simply sets

$$
r(x)=a(x), \quad p(x)=b^{\prime}(x)-c(x),
$$

and recalls equation (1.5).

3. Functionals with two end points singular. The analysis in ML $[1$, p. $279 \mathrm{ff}$.] will make it quite clear how the foregoing analysis may be extended to $J$ when both $x=0$ and $x=b$ may be singular points. The reader's attention is directed particularly to Theorem 11.3 of that paper.

4. Focal points of the $y$-axis. In this section we shall extend the concept of the focal point of a line (cf. §2) $x=x_{0}>0$ to include the line $x=0$ in the case that $p(x)$ is of one sign near $x=0$. In doing this we have two ends in view. We shall find that this extension is of use not only in the study of a variable end point problem, but also, contrary perhaps to expectation, in the study of the fixed end point problem we met in $\$ 2$ of this paper.

Suppose $p(x)$ is of fixed sign on $(0, e)(e>0)$. If a line $x=x_{0}\left(0<x_{0}<e\right)$ has a focal point $a$ on $\left(x_{0}, h\right)$ then as $x_{0}$ tends to zero $a$ increases or decreases steadily according as $p(x)<0$ or $>0$. If $a_{1}=\lim _{x_{0} \rightarrow 0} a$ lies on $[0, h)$, we term $a_{1}$ the focal point of the $y$-axis. If every line $x=x_{0}$, where $x_{0}>0$ is small, fails to have a focal point on $\left(x_{0}, h\right)$, the $y$-axis is said to have no focal point on $[0, h)$. When $a_{1}=0$, the $y$-axis is said to contain its focal point. It will be observed, in particular, that the $y$-axis contains its focal point when $x=0$ is its own first conjugate point.

In the following example there is no point conjugate to $x=0$ on any interval $[0, h)$. Nevertheless the $y$-axis contains its focal point.

EXAmple 4.1. The E. E. of the functional

$$
J=\int_{0}^{b}\left[\frac{1}{x^{2}} y^{\prime 2}-\frac{2}{x^{4}} y^{2}\right] d x
$$

is

$$
\frac{d}{d x}\left(\frac{1}{x^{2}} y^{\prime}\right)+\frac{2}{x^{4}} y=0 .
$$


Linearly independent solutions are $x^{2}$ and $x$. Since the principal solution $w(x)=x^{2}$ has no positive zeros there are no points conjugate to $x=0$ on any finite interval $[0, h)$. Every solution orthogonal to the line $x=x_{0}>0$ may be written in the form

$$
y=k x\left(x-2 x_{0}\right) .
$$

Thus, the focal point of the line $x=x_{0}$ is the point $x=a=2 x_{0}$. It is clear that in this example $x=0$ contains its focal point.

We come to a principal theorem.

TheOREM 4.1. If $p(x)>0$ near $x=0$, and if the $y$-axis contains its focal point, $J$ does not possess a minimum limit.

We may limit ourselves to the case that $x=0$ is not its own first conjugate point. Suppose $p(x)>0$ on $(0, c)$. Then for $x_{0}>0$ sufficiently small a solution $v(x) \not \equiv 0$ of the E. E. such that $v^{\prime}\left(x_{0}\right)=0$ will vanish at $x=a$ on $(0, c)$. Accordingly, by Lemma 2.4 all solutions which are positive near $x=0$ have positive slopes near $x=0$. Thus, if $w(x)$ is a principal solution, the remark following Lemma 2.5 assures us that

$$
\lim \frac{w^{\prime}(x)}{v^{\prime}(x)}=0 .
$$

As in the proof of Lemma 2.6 we may now show that

$$
\lim \int_{x}^{b} p(x) d x=+\infty .
$$

Theorem 4.1 is then a consequence of Theorem 2.1 .

It is not difficult to ascertain from Example 2.1 that the line $x=0$ may contain its focal point without implying that all solutions pass through the origin.

The focal point solution. Suppose $x=0$ is not its own first conjugate point and let $u(x)$ and $v(x)$ be any two linearly independent solutions of the E. E. From (2.10) we see that

$$
\frac{d}{d x}\left(\frac{u^{\prime}(x)}{v^{\prime}(x)}\right)=\frac{c p(x)}{\left[r(x) v^{\prime}(x)\right]^{2}}
$$

$$
(c \neq 0)
$$

Since $p(x)$ is of fixed sign near $x=0$, the ratio $u^{\prime} / v^{\prime}$ tends to a definite limit $L$, finite or infinite, as $x$ tends to zero. If $L$ is finite, let $z(x)=u(x)-L v(x)$; if $L$ is not finite, set $z(x)=v(x)$. We term the solutions $c z(x)(c \neq 0)$ focal point solutions.

LEMMA 4.1. If $z(x)$ is a focal point solution and $y(x)$ is any solution linearly independent of $z(x)$, then 


$$
\lim \frac{z^{\prime}(x)}{y^{\prime}(x)}=0 .
$$

To prove the lemma we distinguish three cases according as $L=0, \infty$, or is finite and nonzero. In the first case take

$$
z(x)=u(x), \quad y(x)=c_{1} u(x)+c_{2} v(x) \quad\left(c_{2} \neq 0\right) .
$$

Then

$$
\frac{z^{\prime}}{y^{\prime}}=\frac{u^{\prime}}{c_{1} u^{\prime}+c_{2} v^{\prime}}=\frac{u^{\prime} / v^{\prime}}{c_{1} u^{\prime} / v^{\prime}+c_{2}},
$$

and $\lim z^{\prime} / y^{\prime}=0$ in this case. The case $L=\infty$ may be handled in precisely analogous fashion with $u$ replaced by $v$ and $c_{1} \neq 0$. We conclude with the case when $L$ is finite and $\neq 0$. Here

$$
\frac{z^{\prime}}{y^{\prime}}=\frac{u^{\prime}-L v^{\prime}}{c_{1} u^{\prime}+c_{2} v^{\prime}}=\frac{u^{\prime} / v^{\prime}-L}{c_{1} u^{\prime} / v^{\prime}+c_{2}} .
$$

The last fraction above clearly tends to zero except possibly when $c_{1} L+c_{2}=0$. But if this last relationship holds, $y=c_{1} z$, contrary to hypothesis. Thus, $\lim z^{\prime} / y^{\prime}=0$ in all cases and the lemma is proved.

Lemma 4.2. Let $p(x)<0$ on $(0, e)$ and let $x_{0}$ and $x_{1}$ be numbers such that $0<x_{0}<x_{1}<e$. If $y_{0}(x)$ and $y_{1}(x)$ are nonnull solutions of the $E$. E. such that $y_{0}^{\prime}\left(x_{0}\right)=0, y_{1}^{\prime}\left(x_{1}\right)=0$, and if $x_{1}-x_{0}$ is chosen sufficiently small,

for $x$ on $\left[x_{0}, x_{1}\right]$.

$$
\frac{y_{0}^{\prime}(x)}{y_{0}(x)}>\frac{y_{1}^{\prime}(x)}{y_{1}(x)}
$$

Let $x_{1}-x_{0}$ be taken small enough that $y_{0}(x) y_{1}(x) \neq 0$ on $\left[x_{0}, x_{1}\right]$. Without loss in generality we may suppose $y_{0}(x)$ and $y_{1}(x)$ to be positive on this interval. The conclusion of the theorem is then equivalent to the condition $\Delta(x)=y_{1}(x) y_{0}^{\prime}(x)-y_{0}(x) y_{1}^{\prime}(x)>0$ on $\left[x_{0}, x_{1}\right]$. It is sufficient to prove that $\Delta\left(x_{0}\right)>0$, or, consequently, that $y_{1}^{\prime}\left(x_{0}\right)<0$. When $y_{0}(x)$ vanishes at some point $x=a>x_{1}$, this conclusion follows from Lemma 2.3 and a classical Sturm comparison theorem. We may, if necessary, however, alter $p(x)$ in a continuous fashion for values of $x \geqq x_{1}$ in such a fashion that $y_{0}(x)$ does vanish at some point $x=a>x_{1}$. It follows that $y_{1}^{\prime}\left(x_{0}\right)<0$ and, hence, that the lemma is true, since the value of $y_{1}^{\prime}\left(x_{0}\right)$ is independent of the described alteration of $p(x)$.

Theorem 4.2. Let $p(x)<0$ near $x=0$ and suppose $z(x)$ is a focal point solution. If $x=\alpha$ is the focal point of the $y$-axis, then $\alpha>0$ and $z(\alpha)=0$. Conversely, the smallest positive zero $\alpha$ (when it exists) of $z(x)$ is the focal point of the $y$-axis. 
Note first that when $p(x)<0$ near $x=0$, the $y$-axis cannot contain its focal point; otherwise, $J$ would not possess a minimum limit on any interval $[0, e]$, no matter how small. $J$, however, evidently does possess a minimum limit on any closed interval throughout the interior of which $p(x)<0$. Thus, if $x=\alpha$ is the focal point of the $y$-axis, $\alpha>0$. We shall show next that $z(\alpha)=0$. Suppose $v(x)$ is a solution of the E. E. linearly independent of $z(x)$. The solution

$$
y(x)=z(x)-\frac{z^{\prime}\left(x_{0}\right)}{v^{\prime}\left(x_{0}\right)} v(x)
$$

is $\not \equiv 0$, and $y=y(x)$ is orthogonal to the line $x=x_{0}$. Further

$$
\lim _{x_{0} \rightarrow 0} y(x)=z(x) .
$$

Suppose $x=a$ is the smallest positive zero greater than $x_{0}$ of $y(x)$ when $x_{0}$ is taken near $x=0$. Since by hypothesis the focal point $x=\alpha$.of the $y$-axis exists,

$$
\lim _{x_{0} \rightarrow 0} a=\alpha,
$$

and because $y(a)=0$ and $z(x)$ is continuous at $x=\alpha$,

$$
0=\lim _{x_{0} \rightarrow 0} y(a)=\lim _{x_{0} \rightarrow 0} z(\lim a)=z(\alpha) .
$$

Thus, the focal point, when it exists, is given by a positive zero of $z(x)$. This zero must be the smallest positive root of $z(x)=0$; otherwise, there would be a conjugate point of $x=0$ on $(0, \alpha)$, and hence, the focal point of $x=0$ would not be $x=\alpha$.

Conversely, suppose $\alpha$ is the smallest positive zero of $z(x)$. We shall show that $x=\alpha$ is the foral point of the $y$-axis. In view of the earlier portion of the proof it will be sufficient to demonstrate the existence of the focal point of the $y$-axis.

To that end let $y=y(x) \not \equiv 0$ be a solution of the E. E. orthogonal to the line $x=x_{0}$ and such that $y\left(x_{0}\right)>0$. We may suppose without loss of generality that $z(x)>0$ on $(0, \alpha)$ and that $p(x)<0$ on $(0, e)(0<e<\alpha)$. If $0<x_{0}<e$, then $y^{\prime}(x)$ does not change sign on $\left(0, x_{0}\right)$. We shall show first that $y^{\prime}(x)<0$ on $\left(0, x_{0}\right)$. Suppose the contrary. A solution $y=u(x) \not \equiv 0$ orthogonal to a line $x=x_{1}$ slightly to the left of $x=x_{0}$ would then have the property that

$$
0=\frac{u^{\prime}\left(x_{1}\right)}{u\left(x_{1}\right)}<\frac{y^{\prime}\left(x_{1}\right)}{y\left(x_{1}\right)} .
$$

On the other hand it follows from Lemma 4.2 that

$$
\frac{u^{\prime}\left(x_{1}\right)}{u\left(x_{1}\right)}>\frac{y^{\prime}\left(x_{1}\right)}{y\left(x_{1}\right)} \text {. }
$$


From this contradiction we infer that $y^{\prime}(x)<0$ on $\left(0, x_{0}\right)$. Thus $y(x)>0$ on this interval. Consider the difference

$$
D(x)=\frac{z^{\prime}(x)}{y^{\prime}(x)}-\frac{z(x)}{y(x)},
$$

and recall that $\lim z^{\prime}(x) / y^{\prime}(x)=0$. If $D(x)>0$ near $x=0$, then $\lim z(x) / y(x)$ $=0$, since $z(x) / y(x)>0$ near $x=0$. Consequently, $z(x)$ is then a principal solution of the E. E., and $y(x)$ must vanish on $\left(x_{0}, \alpha\right)$. If, however, $D(x)<0$ near $x=0$, we have

$$
\frac{z^{\prime}(x)}{z(x)}-\frac{y^{\prime}(x)}{y(x)}>0
$$

near $x=0$. It follows that in this case also $y(x)$ must vanish on $\left(x_{0}, \alpha\right)$. Thus, the focal point of the $y$-axis has been shown to exist, and the theorem follows.

The study of the focal point solution in the case when $p(x)>0$ near $x=0$ requires a somewhat modified treatment. It will be recalled in this case that even when $x=0$ is not its own first conjugate point, the $y$-axis may contain its focal point.

According to Theorems 2.1, 2.2, and 4.1, if $x=0$ is not its own first conjugate point, the $y$-axis contains its focal point if and only if

$$
\lim \int_{e}^{b} p(x) d x=+\infty .
$$

In the theorem which follows we then confine ourselves to the case when this limit is finite.

Theorem 4.3. If $p(x)>0$ near $x=0$, if $x=0$ is not its own first conjugate point, and if

$$
\lim \int_{e}^{b} p(x) d x
$$

is finite, a focal point $x=\alpha$ of the $y$-axis is the smallest positive zero of a focal point solution $z(x)$ and the smallest positive zero of a focal point solution is the focal point of the $y$-axis.

The proof that the focal point $x=\alpha$ of the $y$-axis is the smallest positive zero of $z(x)$ may be given exactly as in Theorem 4.2. Suppose then that $\alpha$ is the smallest positive zero of $z(x)$. We shall show that $x=\alpha$ is the focal point of the $y$-axis. In view of the first portion of the proof it is sufficient to establish the existence of a focal point of the $y$-axis.

To that end suppose that $z(x)>0$ on $(0, \alpha)$ and let $y(x)$ be a solution of the E. E. such that $y\left(x_{0}\right)>0$ and $y^{\prime}\left(x_{0}\right)=0$, where $x_{0}$ is some point on an interval $(0, e)$ where $p(x)>0$. By an argument now familiar one can prove readily that 
$y^{\prime}(x)>0$ on $\left(0, x_{0}\right)$. Further $z^{\prime}(x)<0$ near $x=0$. For, the wronskian $\Delta_{1}(x)$ $=z(x) y^{\prime}(x)-z^{\prime}(x) y(x)>0$ at $x=\alpha$ and, thus, $\Delta_{1}\left(x_{0}\right)=-z^{\prime}\left(x_{0}\right) y\left(x_{0}\right)>0$. It follows that $z^{\prime}\left(x_{0}\right)<0$. Since $x_{0}$ was arbitrary, the conclusion follows. Next, let $u(x)$ be a solution of the E. E. defined by the conditions $u(\alpha+e)=0, u^{\prime}(\alpha+e)$ $=-1$, where $e$ is positive and small. Then $u(x)$ is linearly independent of $z(x)$, and $y(x)$ may be taken as

$$
y(x)=u^{\prime}\left(x_{0}\right) z(x)-z^{\prime}\left(x_{0}\right) u(x) .
$$

Since $\Delta(\alpha)=u^{\prime}(\alpha) z(\alpha)-z^{\prime}(\alpha) u(\alpha)>0$, it follows that $y\left(x_{0}\right)=\Delta\left(x_{0}\right)>0$.

We shall show that either $u(x)$ vanishes at some point $x_{1}$ of $(0, \alpha+e)$ or $u^{\prime}(x)>0$ near $x=0$. In either case the existence of the focal point of the $y$-axis may be readily verified. Suppose then $u(x)>0$ on $(0, \alpha+e)$ and that $u^{\prime}(x)<0$ near $x=0$. Since $\lim z^{\prime} / u^{\prime}=0$, either

$$
\lim \frac{z}{u}=0
$$

or

$$
z / u-z^{\prime} / u^{\prime}>0
$$

near $x=0$. But (4.2) is impossible, since then $z$ would be a principal solution and $u$ would vanish on $(0, \alpha)$. Under the hypotheses of our argument $u^{\prime} / z<0$ near $x=0$ and it follows from (4.3) that

$$
u^{\prime} / u<z^{\prime} / z
$$

near $x=0$. Thus $u$ must vanish on $(0, \alpha)$. From this contradiction we infer the truth of the theorem.

That a focal point solution may or may not be a principal solution is readily seen from examples. Example 5.1 displays a case in which the two are the same. The functional $J$, when $r=p=1$, provides a simple case where the focal point solutions are $c \cos x$, while the principal solutions are $c \sin x$.

5. One end point variable. We consider $J$ as defined in (2.2). A function $y(x)$ and the curve $y=y(x)$ will be termed $F$-admissible [ML, 1, p. 276] on $[0, b]$ if

1. $y(x)$ is continuous on $(0, b]$ and $y(b)=0$;

2. $y(x)$ is absolutely continuous and $y^{\prime 2}(x)$ is integrable Lebesgue on each closed subinterval of $(0, b]$.

It is clear that $A$-admissible curves are also $F$-admissible. We seek conditions under which

$$
\left.\lim \inf J(y)\right|_{e} ^{b} \geqq 0
$$

for all $F$-admissible curves. When this is true we shall say that $J$ possesses an F-minimum limit. 
We come to a necessary condition.

Theorem 5.1. If $p(x)>0$ near $x=0$ and if $J$ possesses an $F$-minimum limit there can be no focal point of the line $x=0$ on the interval $(0, b)$.

Suppose, on the contrary, that the $y$-axis has a focal point on the interval $[0, b)$. Then for $e$ positive and sufficiently small the line $x=e$ would determine a focal point $x=a$ on $(0, b)$. The nonsingular theory will in that event assure the existence of a curve $y=y(x)$ of class $D^{1}$ joining a point $x=a+\epsilon(|\epsilon|$ small $)$ of the $x$-axis to the line $x=e$ along which $J<0$. Along the broken curve defined by the relations

$$
\begin{aligned}
y & =y(e) \\
& =y(x) \\
& =0
\end{aligned}
$$

$$
\begin{array}{r}
(0 \leqq x<e) \\
(e \leqq x \leqq a+\epsilon) \\
(a+\epsilon<x \leqq b)
\end{array}
$$

$J$ also assumes a negative value. The theorem is proved.

Sufficiency conditions; the Hilbert integral. The Hilbert integral associated with $J$ is the line integral

$$
H=\int-\frac{y^{2}}{u(x)^{2}}\left[r(x) u^{\prime}(x)^{2}+p(x) u(x)^{2}\right] d x+2 r(x) y \frac{u^{\prime}(x)}{u(x)} d y,
$$

where $u(x)$ is the solution of the E. E. determined by the conditions $u(b)=0$, $u^{\prime}(b)=-1$. If there is no point conjugate to $x=0$ on $[0, b)$ the coefficients are well-defined continuous functions of $x$ throughout the region $S$ defined by the inequalities $0<x<b,-\infty<y<+\infty$. Denote by $S_{b}$ the point set sum of $S$ and the point $(b, 0)$, and let $g$ be a curve in $S_{b}$ of the form

$$
x=x(t), \quad y=y(t) \quad\left(t_{1} \leqq t \leqq t_{2}\right),
$$

where $x(t)$ and $y(t)$ are absolutely continuous, and $x\left(t_{2}\right)=b, y\left(t_{2}\right)=0$. If $g$ lies in $S$, the Hilbert integral exists and depends only on the end points of $g$. If $g$ terminates at the point $(b, 0), H$ still exists and depends only on its first end point provided $y(t) / u[x(t)]$ is bounded as $t \rightarrow t_{2}[\mathrm{ML}, 1, \mathrm{p} .257 \mathrm{ff}$.].

One may readily verify, as is done in ML (p. 260), that if $y(x)$ is F-admissible on $(0, b]$ and of class $C^{1}$ neighboring $x=b$, if there is no point conjugate to $x=0$ on $[0, b)$, then

$$
\left.J(y)\right|_{e} ^{b}=-r(e) \frac{u^{\prime}(e)}{u(e)} y^{2}(e)+\int_{e}^{b} r(x)\left[y^{\prime}(x)-p(x, y(x))\right]^{2} d x
$$

$$
(0<e<b)
$$

where $p(x, y)=y u^{\prime}(x) / u(x)$.

We are prepared to prove the following result.

Theorem 5.2. If $p(x)>0$ near $x=0$ and if the line $x=0$ has no focal point 
on the interval $[0, b), J$ possesses an F-minimum limit.

Observe that there can be no conjugate point to $x=0$ on $[0, b)$. For suppose such a point $b^{\prime}$ existed. Then for $e>0$ chosen sufficiently small all solutions orthogonal to lines $x=x_{0} \quad\left(0<x_{0}<e\right)$ would have a zero preceding $\left(b+b^{\prime}\right) / 2$. If $e$ were chosen so small that also $p(x)>0$ on $(0, e)$, the line $x=0$ would have a focal point preceding $\left(b+b^{\prime}\right) / 2$, contrary to hypothesis. Therefore, the solution $u(x)$ of the E. E. employed in setting up the Hilbert integral is positive on $(0, b)$. Since $u^{\prime}(x)$ is of fixed sign near $x=0$, that sign must be negative. If this were not true, one could construct a solution $v(x) \cdot$ determined by the conditions $v(c)=u(c), v^{\prime}(c)=0$, where $p(x)>0$ on $(0, e)$ and $0<c<e$. By elementary Sturm theory $v(x)$ would then have a first zero $x=c_{1}$ on $(c, b)$. According to Lemma $2.3, \lim _{c \rightarrow 0} c_{1}<b$, contrary to hypothesis. The theorem now follows from (5.3).

THEOREM 5.3. If $p(x)<0$ near $x=0$, and if the line $x=0$ has no focal point on $[0, b], J$ possesses an F-minimum limit.

It follows from the definition of the focal point of the line $x=0$ that there is no focal point of this line on a slightly longer interval $[0, b+\epsilon)$. Using evident changes one argues as in Theorem 5.2 that there is no point conjugate to $x=0$ on $[0, b+\epsilon)$. We shall show that the derivative $u^{\prime}(x)$ of the solution $u(x)$ used in setting up the Hilbert integral must be less than 0 near $x=0$, and the theorem will then follow from (5.3). Suppose that $u^{\prime}(x)>0$ near $x=0$. Then every solution orthogonal to a line $x=x_{0}$ near $x=0$ must vanish prior to $x=b$; consequently, there would exist a focal point $a$ of the line $x=0$, where $a \leqq b$. From this contradiction we infer the truth of the theorem.

The last theorem can be extended to cover the case when $x=b$ is the focal point of the line $x=0$ by means of a well known device [cf. ML, p. 265]. Suppose $y=y(x)$ is an arbitrary $F$-admissible curve. We wish to show that

$$
\left.\liminf _{e \rightarrow 0} J(y)\right|_{e} ^{b} \geqq 0 .
$$

Let $\mu$ be a number on $(0,1)$ and note that $y_{\mu}(x)=y(x / \mu)$ is $F$-admissible and that by the preceding theorem,

$$
\left.\liminf _{e \rightarrow 0} J\left(y_{\mu}(x)\right)\right|_{\bullet} ^{\mu b} \geqq 0
$$

for each value of $\mu$. Thus, if the limit (5.5) is finite

$$
\left.\liminf _{\mu \rightarrow 1} \liminf _{e \rightarrow 0} J\left(y_{\mu}(x)\right)\right|_{e} ^{\mu b}=\left.\liminf _{e \rightarrow 0} J(y(x))\right|_{e} ^{b} \geqq 0 .
$$

If the limit (5.5) is $+\infty$, 


$$
\left.\liminf _{e \rightarrow 0} J(y)\right|_{e} ^{b}=+\infty .
$$

Example 5.1. Consider the functional

$$
J=\int_{0}^{\alpha}\left[y^{\prime 2}-\left(1-\frac{2}{x^{2}}\right) y^{2}\right] d x
$$

where $\alpha$ is the smallest positive root of the equation $x=\tan x$. One observes that $p(x)<0$ near $x=0$ and that $p(x)$ changes sign on the interval of integration. A principal solution of the E. E. is

$$
w(x)=\frac{\sin x}{x}-\cos x .
$$

A solution linearly independent of $w(x)$ is

$$
u(x)=\sin x+\frac{\cos x}{x} .
$$

It is readily verified that $x=\alpha$ is the focal point of the $y$-axis and that $\alpha$ is the smallest positive zero of $w(x)$. Computation will show that the singularity function is negative near $x=b$. However, an appeal to the extension of Theorem 5.3 indicates that (5.4) holds for all $F$-admissible functions $y(x)$.

The case $p(x) \equiv 0$. If $p(x) \equiv 0$ on $(0, e], J$ evidently possesses a minimum limit on this interval. It follows from Theorem 5.3 of ML that $J$ possesses a minimum limit over a longer interval $[0, b]$ if there is no point conjugate to $x=0$ on $[0, b)$, for there is clearly a family of solutions of the E. E. which near $x=0$ take the form $y=$ constant.

The notion of the focal point of the $y$-axis is readily extended to this case. One observes that the $y$-axis never contains its focal point, and that $J$ possesses an $F$-minimum limit over any interval $[0, b]$ such that $[0, b)$ contains no focal point of the $y$-axis.

\section{BiBLIOGRAPHY}

Bliss, Gilbert A.

1. Lectures on the calculus of variations, University of Chicago Press, 1946. Bôcher, MAXIME

1. Leçons sur les méthodes de Sturm, Paris, Gauthier-Villars, 1917.

MORSE, MARSTON

1. The calculus of variations in the large, Amer. Math. Soc. Colloquium Publications, vol. 18, New York, 1934.

Morse, Marston and Leighton, Walter

1. Singular quadratic functionals, Trans. Amer. Math. Soc. vol. 40 (1936) pp. 252-286.

WASHINGTON UNIVERSITY,

St. Louis, Mo. 